\title{
Isatis indigotica: from (ethno) botany, biochemistry to synthetic biology
}

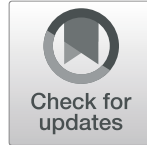

Jingxian Feng ${ }^{1}$, Doudou Huang ${ }^{1}$, Yingbo Yang ${ }^{1,2}$, Junfeng Chen' ${ }^{1}$, Shi Qiu', Zongyou Lv¹, Xueqi Ma', Yuanyu Li', Rongrong $\mathrm{Li}^{1}$, Ying Xiao ${ }^{1 *}$ and Wansheng Chen ${ }^{1,3^{*}}$ (i)

\begin{abstract}
Isatis indigotica Fort. (Chinese woad) is a species with an ancient and well-documented history as an indigo dye and medicinal plant. It is often confused with Isatis tinctoria L. (European woad), a medicinal plant in Europe. Here, the differences between I. indigotica and I. tinctoria are systematically described. The usage development history, clinical applications and pharmacological activities, and chemical components of I. indigotica are also summarized. Lignans, indole alkaloids, and their corresponding derivatives have been identified as the major active ingredients of I. indigotica and are associated with anti-viral, anti-inflammatory, anti-cancer, and other health-promoting activities. Notable progress has been made in understanding the biosynthetic pathway and regulation mechanism of lignans and indole alkaloids in I. indigotica, the results from which should facilitate the process of targeted metabolic engineering or synthetic biology. Moreover, multiple biotechnology methods such as polyploid breeding and genetic engineering have been used with I. indigotica to result in, for example, greater yields, higher levels of bioactive component accumulation, and enhanced stress tolerance to salt, drought, and insects. Some issues require additional analyses, and suggestions for future research on I. indigotica are also discussed.
\end{abstract}

Keywords: Isatis indigotica, Lignans, Indole alkaloids, Metabolic engineering, Synthetic biology

\section{Introduction}

Isatis indigotica Fort. is a biennial herbaceous plant belonging to the Cruciferae (Brassicaceae) family and has a long history as one of the most prevalent Chinese medicinal herbs and dyes. To this day it continues to be used in medical treatments, the pharmaceutical industry, and the handmade textile industry. The root and leaf of I. indigotica are used to prepare three different traditional Chinese medicines. The dry roots, known as Banlangen (ISATIDIS RADIX), have anti-inflammatory and anti-viral activities, and can be further processed into Banlangen Keli. Both of these preparations are widely used for the

\footnotetext{
* Correspondence: xiaoyingtcm@shutcm.edu.cn;

chenwansheng@shutcm.edu.cn

${ }^{1}$ Research and Development Center of Chinese Medicine Resources and Biotechnology, The Ministry of Education (MOE) Key Laboratory for

Standardization of Chinese Medicines, Institute of Chinese Materia Medica, Shanghai University of Traditional Chinese Medicine, Shanghai 201203, China Full list of author information is available at the end of the article
}

treatment of flu and eruptive epidemic diseases in China (Zhang et al., 2016c). The dry leaves, called Daqingye (ISATIDIS FOLIUM), have pharmacological activity similar to that of Banlangen and are widely used for the treatment of fever, epidemic parotitis, and pharyngitis. Fufang Daqingye Heji, a liquid that is consumed orally, is the main preparation of Daqingye (Zhu et al., 2004). Qingdai (INDIGO NATURALS) is a more processed product of the leaves. It is the dry foam of fermented leaves and has a dark blue color, and is applied to the skin and used to heal wounds, acne, erysipelas, and carbuncles (Chen, 2013a).

Interestingly, the original plant species used for these three kinds of medicines did not consist solely of I. indigotica. Over time, I. indigotica gradually became the main cultivated species, mainly because of its larger output of materials and its better pharmacological activities. Currently, it is the only remaining species among the plants originally used to produce Banlangen and 
Daqingye and is the main plant component of Qingdai in the Chinese Pharmacopoeia (2020).

Isatis tinctoria L. (European woad) is a species in the same genus as I. indigotica (Chinese woad). The use of $I$. tinctoria in Europe also has a centuries-old history. Celtic and Germanic people used I. tinctoria as a source of indigo for dyeing and as a prophylactic in Roman times. From the 12th to the seventeenth century, I. tinctoria was widely promoted in Germany, France, England, and Italy (Hamburger, 2002; Speranza et al., 2020). Not until 2011, however, was it finally recognized as a medicinal plant in Europe (European Pharmacopoeia, 2011). Because of the similar uses and appearances of these two plants there has, however, been a long-standing dispute about the classification of I. indigotica and I. tinctoria. Some researchers have considered I. indigotica to be a synonym of $I$. tinctoria, although some taxonomists have confirmed that these two species have many differences in their morphology, genetics, and physiology (Angelini et al., 2007; Danz et al., 2001; Sun and Pang, 2002, 2013).

According to modern pharmacological investigations, I. indigotica possesses various biological activities, including its extracted parts and compounds. The active ingredients can be divided into several major groups; lignans and indole alkaloids and their corresponding derivatives, flavones, glycosides, and polysaccharides. A better understanding of these compounds and their biosynthetic pathways will extend our knowledge of the chemical characteristics of I. indigotica.

This review aims to clarify the recorded history of how I. indigotica gradually became the main plant in its related herbal preparations, to show the taxonomist's point of view with respect to distinguishing $I$. indigotica from I. tinctoria, and to summarize the advanced knowledge of the pharmacologically-active secondary metabolites of I. indigotica. The biosynthetic pathways and key regulatory genes involved in the two major active ingredients, lignans and indoles, as well as biotechnology methods to improve I. indigotica, are reviewed and discussed.

\section{Historical use of $I$. indigotica}

China is rich in plant species. The historical record shows that several species have been used for blue (indigo) dyeing, while also showing detoxifying activities. Such plants were referred to as Lan (indigo plant) in ancient China. These include Liaolan (Polygonum tinctorium) (Han, 2005; Jia et al., 1996), Songlan (I. indigotica) (Anonymous, 2008), Malan (Baphicacanthus cusia) (Su et al., 1994), and Mulan (Indigofera tinctoria) (Chen, 2004; Wang et al., 2020). The current Chinese Pharmacopeia describes Daqingye as the dry leaves of $I$. indigotica; Banlangen as dry roots; and Qingdai as the dry powder, agglomerates, or granules obtained by processing the aerial parts of I. indigotica, P. tinctorium, and B. cusia (Chinese Pharmacopoeia, 2020). The recorded components of these three traditional Chinese medicines have, however, changed over time.

A record of the medicinal use of Lan first appeared in the "Shen Nong Ben Cao Jing" ("Shen Nong's Herbal Classic") in the Spring and Autumn Period (770-475 $\mathrm{BCE})$ (Anonymous, 2008). It is stated therein that the seeds of Lan, i.e. Lanshi, have the effect of detoxification. Huang and Chen (1965) reported that the Lan referred to in that ancient text was I. indigotica. In addition, $I$. indigotica was cultivated in the Late Eastern Han dynasty (?-CE 220) (Huang and Chen, 1965).

By the Wei, Jin, and Northern and Southern dynasties (CE 220-589), the leaves of some indigo plants with their seeds were included as antidotes for poison or insect poison. For this use, the juice processed from young leaves was the better choice (Tao et al., 2013; Wang et al., 2020). It should be noted here that the leaves used were just the leaves of Lan and were not specifically named Daqingye.

Daqing (Clerodendrum cyrtophyllum Turcz.) is a species from the family Verbenaceae. Daqingye was first used as the term to refer to a preparation of its leaves in ancient China; this preparation had similar efficacy to that of Lan and was used to treat aphtha. In the Qing Dynasty (CE 1636-1912), Yang Shitai, a famous physician, wrote in his book that Lan or Qingdai could be used as a substitute for Daqing when the latter was insufficient (Yang et al., 2009). From then on, Daqing and Lan were used interchangeably for medical use. Because of its greater curative effects and the larger amount of plant material it produced, Lan gradually replaced Qaqing as the main plant component of this medicine, although the name Daqingye was not changed (Wang et al., 2020; Yang et al., 2009).

Reference to Banlangen first appeared in the officially published books of traditional Chinese medicine in the Song dynasty (CE 960-1279), in which it was described as the root of Lan and having the same efficacy as the leaf. Banlangen became a kind of commonly used medicine in the Yuan dynasty (CE 1271-1368). The first original plant used to produce Banlangen was Malan ( $B$. cusia), a specialty plant in Fujian province in China, where it was referred to as Banlan in the Fujian dialect. Therefore, the processed product derived from its root is referred to as Banlangen. During the late Qing dynasty, however, the original plant species used to produce Banlangen was confused with various Lan species (Huang and Chen, 1965; Teng, 1996). "Zhong Hua Ben Cao" ("Chinese Materia Medica") documented Songlan (I. indigotica) as the original plant of Banlangen, and at that time I. indigotica had become the main cultivated species. Currently, the Chinese Pharmacopoeia (2020) 
defines Nanbanlangen (southern Banlangen) as the dry root and rhizome of Malan (B. cusia)

Qingdai originally referred to the powder extracted from shells imported from Persia, which also has a detoxifying effect, albeit with very low yield. It was discovered that the dry foam in the indigo dye vats had an effect similar to that of the powdered shell, and thus the dry foam came to replace the shell powder and also assumed its name (Teng, 1996).

Over the period of several dynasties it was determined that $I$. indigotica was indeed the best cultivated species for these medicines (Huang and Chen, 1965; Teng, 1996; Wu and Wang, 1996). This is a manifestation of the expression "practice is the only criterion for testing truth."

\section{Differences between I. indigotica and I. tinctoria}

Taxonomists conducted morphological, cytological, and palynological observations of I. indigotica and I. tinctoria, and analyzed their enzymes and metabolites. They concluded that I. indigotica differs from I. tinctoria and is native to China (Qiao, 1984; Wang and Zhou, 1982) (Fig. 1).

The seedlings and basal leaves of I. indigotica are light pinkish-gray, smooth, and hairless. The stem leaves are mostly oblong and also smooth and hairless. The petals are oblanceolate, with a claw-shaped base and truncated top. The fruits are oblong-ovate or oblong, wedgeshaped at the base, and blunt round or truncated at the top; their middle ribs are thin and inconspicuous, and some locules do not bulge. This species has seven pollen mother cells and $2 \mathrm{n}=14$ somatic cells (Qiao and Cui, 1982).

In contrast, the seedlings and basal leaves of I. tinctoria are blue, with unicellular, non-glandular hairs. The stem leaves are mostly long and lanceolate, with sparse unicellular, non-glandular hairs. The petals are obovate with an inconspicuous and claw-shaped base and round top. The fruits are mostly wedge-shaped, round, or acuminate at the base; their middle ribs are thin and inconspicuous, and the locules bulge conspicuously. This species has 14 pollen mother cells and $2 \mathrm{n}=28$ somatic cells (Qiao and Cui, 1982).

From the perspective of plant evolution, chromosomes usually change from low to high ploidy level, and less often in the other direction. If the two members of the genus Isatis are in fact the same species, the tetraploid form of I. indigotica should be similar to I. tinctoria. However, tetraploid I. indigotica, when induced by colchicine growth retardation, has wider and thicker leaves, and its flower organs, pollen grains, fruits, and stomata are larger than those of the diploid I. indigotica, with notable differences to I. tinctoria. Therefore, the merging of these two species cannot be supported.

As per their respective metabolites, Huang (2020) detected much higher levels of indole alkaloid derivatives, quinolines, and flavonoids (e.g. isovitexin, isovitexin-3"-

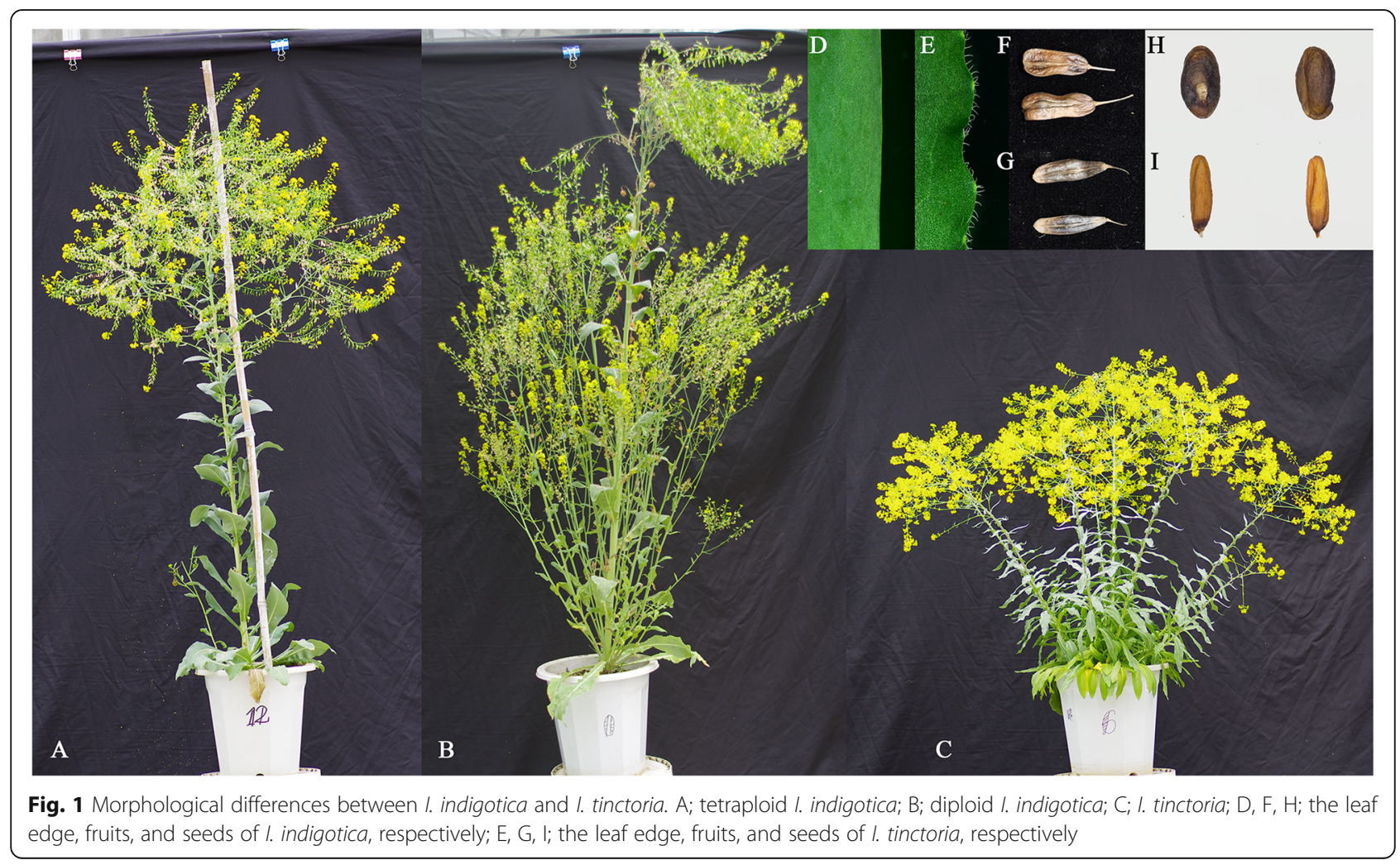


O-glucoside-7-O-glucoside, isovitexin-3"-O-glucoside, isoscoparin-3"-O-sinapoylglucoside, and isoscoparin-3"O-feruloylglucoside) in I. indigotica than that in I. tinctoria. In contrast, the organic acid derivatives accumulated to higher levels in I. tinctoria than I. indigotica. Although one species did not consistently outproduce the other with respect to these metabolites, the differences between these two species were constant.

\section{Clinical applications and pharmacological activities of $I$. indigotica}

As one of the most popular herbal drugs in China, $I$. indigotica has been used most often for detoxification, against fever, and curing aphtha in the three forms of traditional Chinese medicine described above. In modern clinical applications, however, herbal extracts of $I$. indigotica and prescriptions or the isolated bioactive constituents have shown preventive and therapeutic effects against influenza and upper respiratory tract infection, inflammation, and allergies. For instance, a waterbased extract of the roots can inhibit different subtypes of human and avian influenza viruses, including H1N1 and H3N2 (Haruyama and Nagata, 2013; Ho et al., 2014; Yang et al., 2012; Zhang et al., 2020). In combination with other herbal drugs, the leaves and roots of I. indigotica are used to treat icteric hepatitis, parotitis, condyloma, palmoplantar pustulosis, epidemic keratoconjunctivitis, and viral myocarditis (Han, 2012; Li et al., 2007; Ma, 2003).

Numerous pharmacological studies have reported the activities of extracts or isolated compounds of $I$. indigotica both in vivo and in vitro. These preparations or compounds have anti-viral, anti-inflammatory, analgesic, anti-microbial, and even anti-tumor activities. The leaf extracts can inhibit infection and proliferation of influenza A, H7N9, encephalitis B, mumps viruses, HSV-II, Dengue virus II, and Cytomegalovirus, among others (Ma, 2014; Zhou et al., 2006); directly neutralize and degrade endotoxin in actinomycin D-sensitized mice (Shi and Zhang, 2006) and promote interleukin 2 (IL-2) secretion by spleen lymphocytes induced by concanavalin A to enhance immunity in normal mice (Hsuan et al., 2009; Liang et al., 2000). They also have anti-bacterial effects against Staphylococcus aureus and Escherichia coli (Lejars and Hajnsdorf, 1863) and promote bile excretion and relieve pain (Hong et al., 2010; Liu et al., 2009). The root extracts have similar activities, such as inhibiting the human $\mathrm{H} 7 \mathrm{~N} 9$ avian influenza virus by preventing the virus from invading the host cells; inhibiting HSV-1 by preventing its replication and proliferation in cells; inhibiting E. coli, Staphylococcus epidermidis, Pneumococcus, Haemophilus influenzae, and Streptococcus; and decreasing the levels of tumor necrosis factor- $\alpha$ (TNF- $\alpha$ ) and IL-6 in peritoneal macrophages of mice ( $\mathrm{Hu}$ et al.,
2003; Wang et al., 2012). Ghosh et al. (2020) reported two potential SARS CoV-2 Mpro inhibitors, sinigrin and hesperetin, derived from the root of I. indigotica, using molecular docking. These two compounds interacted with the important catalytic residues of Mpro (His41 and Cys145), and might be considered for corona virus disease 2019 (COVID-19) treatment (Ghosh et al., 2020).

Various compounds isolated from I. indigotica have been reported as bioactive components, such as tryptanthrin, indirubin, lariciresinol, clemastanin B, epigotin, and polysaccharides. Tryptanthrin has been found by a few studies to have anti-inflammatory activity and inhibits breast cancer cell proliferation in vitro. It inhibited cyclooxygenase 2 (COX-2) and 5-lipoxygenase (5-LOX) in cell-based assays and inhibited Trichophyton mentagrophytes, which causes tinea pedis (Tang et al., 2004). Zeng et al. (2021) reported that tryptanthrin inhibited the proliferation, migration, and invasion of human breast adenocarcinoma Michigan Cancer Foundation-7 (MCF-7) cells, and regulated the level of related proteins in vitro. Tryptanthrin effectively inhibited tumor growth in $4 \mathrm{~T} 1$ murine breast cancer model; modulated expression levels of nitric oxide synthase 1 (NOS1), COX-2, and nuclear factor kappa-B (NF-kB) in mouse tumor tissues; and regulated some factors such as IL-2, IL-10, and TNF- $\alpha$ in mice (Zeng et al., 2021). Indirubin possesses anti-tumor activity as shown by its ability to inhibit the growth of transplanted tumors and alleviate chronic myeloid leukemia (Xu et al., 2010). Indirubin and its derivates can interrupt virus-induced kinase activation and NF- $\kappa B$ translocation (Ye et al., 2011), and inhibit cyclindependent kinase (CDK) and histone deacetylase (HDAC) in several cancer cell lines (Cao et al., 2021). Li (2015) determined that lariciresinol and its glycosylated products were useful for the treatment of influenza A1 virus. Yang et al. (2013) determined that a lariciresinol derivative, clemastanin $\mathrm{B} \quad\left[7 S, 8 R, 8^{\prime} R\right.$ (-)-lariciresinol-4,4'-bis- $O-\beta$-D-glucopyranoside], can block the translocation of nucleocapsid protein at an early stage of virus replication (Yang et al., 2013). Epigotin has a strong inhibitory effect on influenza A1 virus FM1 by reducing susceptibility to the virus via mitochondrial anti-viral signaling (Luo et al., 2019). Polysaccharides prevent the influenza virus from attaching to host cell surfaces (Yang et al., 2012; Zhao et al., 2008); inhibit Hepatitis B virus (HBV) in vitro by reducing extracellular and intracellular DNA levels of HBsAg, HBeAg, and HBV in HepG2.2.15 cells (Yong and Aisa, 2011; Zhao and Aisa, 2012); and promote specific immunity, nonspecific immunity, humoral immunity, or cellular immunity effects (Grienke et al., 2019). More detailed clinical applications and pharmacological activities of 
I. indigotica were systematically reviewed by Chen et al. (2021a).

\section{Chemical components of $I$. indigotica}

Phytochemical investigations of I. indigotica have led to the isolation of various natural compounds including alkaloids, lignans, flavonoids, phenolic acids, and polysaccharides. Ultra high performance liquid chromatography quadrupole/time of flight-MS/MS (UHPLC-Q/TOF-MS/ MS) studies of I. indigotica identified 116 indole alkaloids and their derivatives, 20 lignans and their derivatives, 105 carbohydrates and carbohydrate conjugates, 45 quinolines, 28 tetrapyrroles, 50 flavonoids, 35 alkaloids and their derivatives, 104 organic acids and their derivatives, and 360 lipid and lipid-like molecules (Huang, 2020). More than 150 of these components have been assessed in phytochemical pharmacology studies. Indole alkaloids and lignans, along with their corresponding derivatives, are considered the two major bioactive ingredients of $I$. indigotica.

Alkaloids in I. indigotica fall into two categories; indole and quinoline alkaloids. Indigo and indirubin, the two main indole alkaloids, have long been used as blue and red dyes, respectively, in China (Lu et al., 2012). Historically, I. indigotica was prepared as a decoction with water; more recently, researchers have used extraction with ethanol and methanol, which resulted in the isolation of several polar indole alkaloids that contained more than one glycoside. Six new diglycosidic indole alkaloid derivatives, referred to as isatigotindolediosides A-F, were isolated from dried roots of I. indigotica (Meng et al., 2017a). Another interesting class of indole compounds was discovered from aqueous extraction of I. indigotica (Meng et al., 2017b), in which there is a free sulfonic acid group. The sulfonic acid group not only increases the water solubility of the compounds but also enhances the anti-viral activity of the plant. As I. indigotica is the source of many mono-indole-related compounds, these compounds with their free indole groups can be naturally polymerized into more-complex indolerelated compounds. In 2012, a previously uncharacterized molecule with linkages between 2-(4-methoxy- $1 \mathrm{H}$ indol-3-yl) acetonitrile and 2-( $1 H$-indol-3-yl) acetonitrile was isolated from I. indigotica root (Chen et al., 2012), which was an enriched indole alkaloid type. Since then, a series of compounds with indole dimers have been isolated and identified from I. indigotica (Liu et al., 2015). Another important alkaloid type is the quinoline alkaloids, which contain a benzopyridine ring. Quinoline compounds in I. indigotica are also diverse and exist both as monomers and as polymers after polymerization with other groups. For example, $(+)-(R)-2$-oxo-1,2,3,4tetrahydroquinoline-4-carboxamide was found to contain a single quinoline group, whereas isatisindigoticanine $\mathrm{B}$ contains a quinoline and an indole group (Zhang et al., 2019a).

Lignan has long been regarded as an effective antiviral component in I. indigotica. Zhong's laboratory reported that the two most common lignans, lariciresinol4-O- $\beta$-D-glucopyranoside and clemastanin $\mathrm{B}$, significantly decrease the pathogenicity of the virus $\mathrm{H} 1 \mathrm{~N} 1$ ( $\mathrm{Li}$ et al., 2015; Yang et al., 2013). In an effort to identify additional bioactive lignans, 17 lignans were identified in an ethanol extract of I. indigotica (Zhang et al., 2019b). Lignans have long been considered to be formed by the formation of a C6-C3 junction between two monomers, although several lignans, consist of three monomer molecules, including isatindigosesquilignans $\mathrm{A}$ and $\mathrm{B}$. It seems that the third monomer was also added by C6-C3 junction on the base of the dimer. The isolation of lignans with different molecular structures will help clarify their synthesis mechanisms.

\section{Biosynthetic pathways and metabolic regulation of bioactive compounds in I. indigotica}

Previous studies based on transcriptomes revealed candidate genes for the biosynthesis of types of active compounds in I. indigotica. Chen et al. (2013b) reported a database of 36,367 isogenes generated by performing transcriptome sequencing of the hairy roots of I. indigotica, which are induced by Agrobacterium tumefaciens; they identified 104 unigenes involved in the general pathways of active compounds. However, the number of identified genes was limited, the expression level of the genes showed only the phenotypes of the various lines, and genes from hairy roots may not display all the characteristics of I. indigotica plants. Kang et al. (2020) reported a chromosome-scale genome assembly of $I$. indigotica with a total size of $293.88 \mathrm{Mb}$ and scaffold $\mathrm{N} 50=36.16 \mathrm{Mb}$. They annotated 30,323 protein-coding genes with high confidence, including 59 genes involved in terpenoid and sterol biosynthesis, 66 genes involved in biosynthesis of lignans and flavonoids, and 32 genes involved in biosynthesis of indole alkaloids. They suggested that numerous genes involved in the biosynthesis of the two major types of active compounds showed increased copy numbers because of tandem duplication, which may drive the production of active compounds.

\section{Biosynthesis and regulation of lignans}

Lignans are a major class of phenylpropanoids, with two phenylpropane units connected through a $\beta, \beta^{\prime}$-linkage, and are a family of secondary metabolites found widely in plants. The phenylpropanoid biosynthetic pathway in plants begins with phenylalanine. The 4-coumaroyl CoA is the bifurcation point of the metabolic flux. Flavonoids, isoflavonoids, phenylpropanoid esters, lignin, and lignans are all formed from this point. Lignin and lignans come 
from the same branch, in which $p$-coumaroyl CoA transfers into three kinds of monolignols, and then numbers of monolignols are converted to lignin. On a separate branch, the dimerization of coniferyl alcohol represents the initiation of lignan biosynthesis, and this branch competes with the lignin biosynthetic branch. One of the two furan nuclei in the pinoresinol structure is opened to generate lariciresinol, and then the other ring is opened to generate secoisolariciresinol. The two free hydroxyl groups reform into a five-membered ring to generate matairesinol. The four lignans in this flux can all be glycosylated with one or two glucose molecules on their free hydroxyl groups (Chen et al., 2021b) (Fig. 2, Table 1).

Phenylalanine ammonia-lyase (PAL) is the first key enzyme in the phenylalanine-derived pathway. Ma et al. (2016) and $\mathrm{Lu}$ et al. (2006a) isolated two PAL genes from I. indigotica, IiPAL1 and IiPAL2; both of the recombinant proteins catalyze the conversion of L-phenylalanine to trans-cinnamic acid. A correlation analysis suggested that IPAL1 is more closely associated with the biosynthesis of secondary metabolites.

Cinnamate 4-hydroxylase $(\mathrm{C} 4 \mathrm{H})$ catalyzes the para position hydroxylation of cinnamic acid into 4-coumaric acid. Hu et al. (2015) cloned IiC4H from I. indigotica. Its full-length sequence is $1647 \mathrm{bp}$ with an open reading frame (ORF) of $1530 \mathrm{bp}$ that encodes a protein of 509 amino acids (aa). Ultraviolet-B (UV-B), methyl jasmonate (MeJA), abscisic acid (ABA), and gibberellic acid $\left(\mathrm{GA}_{3}\right)$ treatments upregulate its transcription to varying degrees (Hu, 2010; Hu et al., 2015).

In the main pathway that generates 4-coumaroyl CoA, 4-coumarate CoA ligase (4CL) is the final enzyme. Three Ii4CL genes were cloned and characterized. Recombinant Ii4CL2 can use 4-coumaroyl acid, caffeic acid, ferulic acid, and sinapic acid as substrates, whereas Ii4CL3 uses only caffeic acid and ferulic acid, and Ii4CL1 does not use any of these four compounds as a substrate. RNA interference (RNAi) of Ii4CL2 in hairy-root lines of $I$. indigotica showed induced accumulation of lariciresinol (maximum of 2.5-fold) relative to that in wild-type lines. In contrast, Ii4CL3 RNAi in hairy-root lines resulted in a lower accumulation of lariciresinol (minimum of 0.4fold) relative to wild-type lines. Therefore, Ii4CL3 plays a significant role in synthesis of lariciresinol (Di et al., 2012; Zhang et al., 2016a).

Coumaric acid 3-hydroxylase $(\mathrm{C} 3 \mathrm{H})$ catalyzes the 3position hydroxylation of 4-coumaroyl $\mathrm{CoA}$ into caffeoyl CoA. Xuan (2012) isolated $\mathrm{IiC} 3 H$ from $I$. indigotica. Its full-length sequence is $1830 \mathrm{bp}$ with an ORF of $1527 \mathrm{bp}$ that encodes a 509 -aa protein. Its transcription is induced by UV and MeJA treatment. Hairy-root lines that overexpress $\mathrm{IiC} 3 \mathrm{H}$ show a fourand nine-fold increase in lariciresinol and sinapyl alcohol, respectively, relative to that in wild-type lines (Chen et al., 2015; Xuan, 2012).

Caffeoyl CoA 3-O-methyltransferase (CCoAOMT) catalyzes the conversion of deoxymethyl of caffeoyl CoA into feruloyl CoA. Li et al. (2021) cloned IiCCoAOMT from I. indigotica; it has a full-length sequence of 1098 bp with an ORF of 774 bp that encodes a 257 -aa protein. Transcription of this gene is induced by low temperature and $\mathrm{NaCl}, \mathrm{MeJA}$, salicylic acid (SA), $\mathrm{ABA}$, and $\mathrm{GA}_{3}$ treatments (Li et al., 2021; Lu, 2006).

Cinnamoyl-CoA reductase (CCR) catalyzes the nicotinamide adenine dinucleotide phosphate (NADPH)dependent reduction of cinnamoyl CoA esters to their corresponding cinnamaldehydes, such as coniferaldehyde and 4-coumarldehyde. Hu et al. (2011) cloned IiCCR, which has a full-length sequence of $1368 \mathrm{bp}$ and an ORF of $1026 \mathrm{bp}$ that encodes a 341-aa protein. Treatment with MeJA, GA ${ }_{3}$, and UV-B induced IiCCR expression with the highest level about 1.5-fold higher than that of the wild type. In contrast, ABA had a repressive effect on IiCCR expression, with a minimum level about 5-fold lower than the wild type.

Cinnamyl alcohol dehydrogenase (CAD) catalyzes the conversion of cinnamyl aldehydes into their corresponding alcohols, such as $p$-coumaryl alcohol, coniferyl alcohol, and sinapyl alcohol. A single IiCAD gene has been cloned; it has a full-length sequence of $1042 \mathrm{bp}$ and an ORF of $1083 \mathrm{bp}$ that encodes a 360-aa protein. Both UV-B and MeJA induce $I i C A D$ expression to varying degrees $(\mathrm{Hu}, 2010)$.

Hydroxycinnamoyl-CoA shikimate/quinate hydroxycinnamoyl transferase (HCT) catalyzes two steps in the phenylpropanoid pathway, from $p$-coumaroyl CoA to $p$ coumaroyl shikimate and from caffeoyl shikimate to caffeoyl CoA in Arabidopsis thaliana. Decreasing the expression of HCT inhibits the accumulation of coniferyl alcohol (Besseau et al., 2007). Dong et al. (2015) isolated IiHCT, the homolog of AtHCT, and showed that its transcriptional $I H C T$ is induced by MeJA treatment in the hairy-root lines of I. indigotica.

Dirigent protein (DIR) catalyzes the coupling of two coniferyl alcohol molecules into pinoresinol. Li et al. (2014) and Chen (2018) identified two IiDIR genes (IiDIR1 and IiDIR2) among the DIR family in I. indigoticathat catalyze the synthesis of (-)pinoresinol. Further studies showed that IiDIR2 promotes the accumulation of $(+)$ secoisolariciresinol.

Pinoresinol/lariciresinol reductase (PLR) is involved in the transformation from pinoresinol to lariciresinol and from lariciresinol to secoisolariciresinol. Three IiPLR genes were isolated from I. indigotica. However, only IiPLR1 was associated with the ability to inhibit accumulation of lariciresinol. The IiPLR1 RNAi hairy-root lines showed decreased lariciresinol levels, whereas hairy-root 


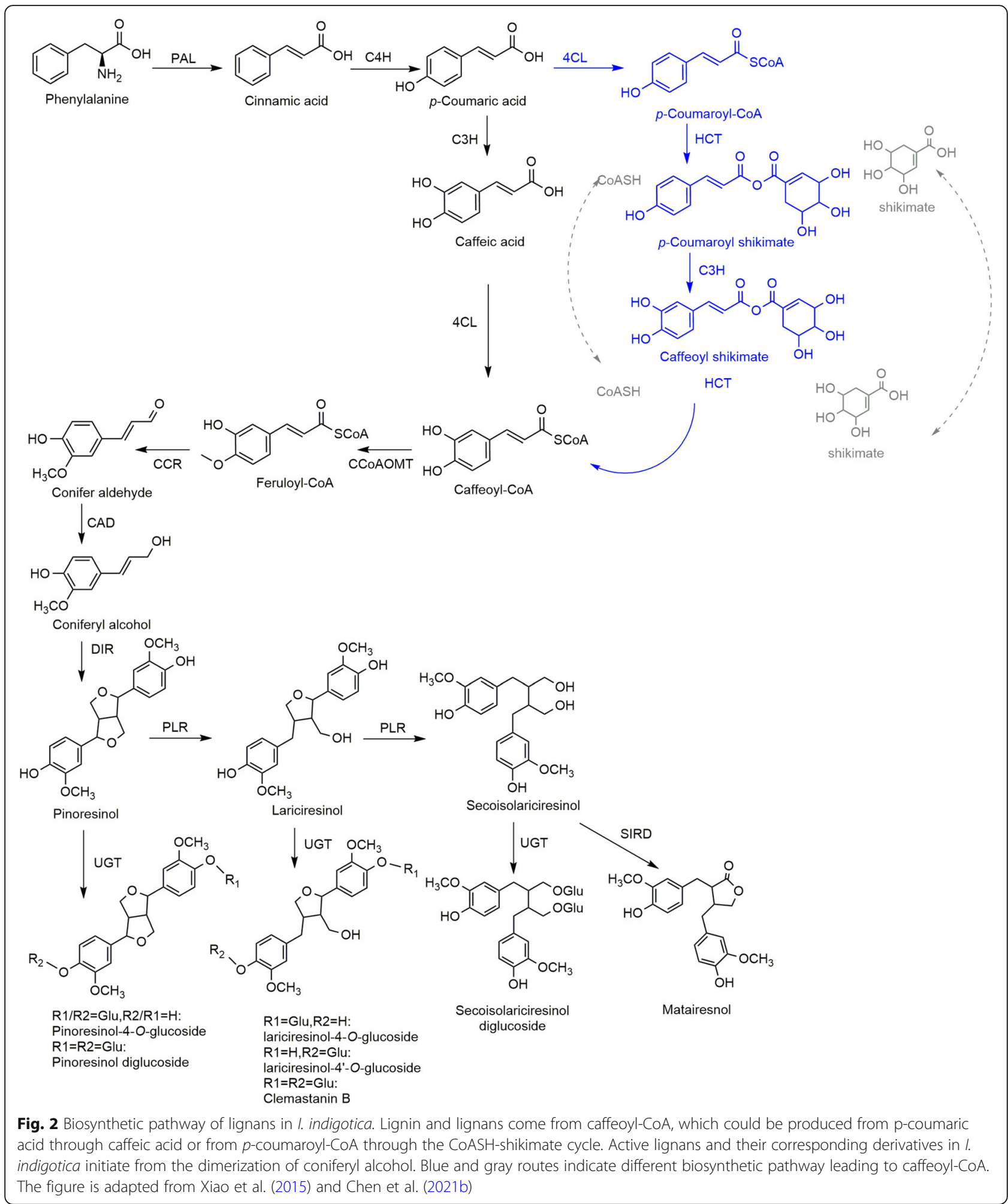

lines that overexpressed this gene showed increased lariciresinol levels. Consistently, recombinant protein IiPLR1 was able to catalyze the target reaction from pinoresinol to lariciresinol and even secoisolariciresinol in vitro (Xiao et al., 2015, 2021). Recently, crystal structures for IiPLR1 in the apo, substrate-bound, and product-bound states were fully elucidated, and the molecular mechanism underlying its substrate specificity 
Table 1 Enzymes involved in lignan biosynthetic pathway of I. indigotica

\begin{tabular}{|c|c|c|c|c|c|}
\hline Name & Enzyme & Gene ID & Substrate & Product & Reference \\
\hline \multirow[t]{2}{*}{ Phenylalanine ammonia-lyase } & PAL1 & - & \multirow[t]{2}{*}{ Phenylalanine } & \multirow[t]{2}{*}{ trans-Cinnamic acid } & \multirow[t]{2}{*}{ Ma et al., 2016} \\
\hline & PAL2 & - & & & \\
\hline Cinnamate 4-hydroxylase & $\mathrm{C} 4 \mathrm{H}$ & GU014562 & Cinnamic acid & 4-Coumaric acid & Hu et al., 2015 \\
\hline \multirow[t]{3}{*}{ 4-Coumarate CoA ligase } & $4 \mathrm{CL}$ & GQ872418 & - & - & Di et al., 2012 \\
\hline & $4 C L 2$ & KC430622 & $\begin{array}{l}\text { 4-Coumaroyl acid; caffeic acid; } \\
\text { ferulic acid; sinapic acid }\end{array}$ & $\begin{array}{l}\text { 4-Coumaroyl CoA; caffeoyl CoA } \\
\text { et al. }\end{array}$ & $\begin{array}{l}\text { Zhang et al., } \\
\text { 2016a }\end{array}$ \\
\hline & $4 \mathrm{CL} 3$ & KC430623 & Caffeic acid; ferulic acid & Caffeoyl CoA; Feruloyl CoA & $\begin{array}{l}\text { Zhang et al., } \\
2016 b\end{array}$ \\
\hline Coumaric acid 3-hydroxylase & $\mathrm{C} 3 \mathrm{H}$ & JF826963 & 4-Coumaroyl CoA & Caffeoyl CoA & Chen et al., 2015 \\
\hline Caffeoyl CoA 3-O-methyltransferase & CCOAOMT & DQ115904 & Caffeoyl CoA & Feruloyl CoA & Li et al., 2021 \\
\hline Cinnamoyl-CoA reductase & $C C R$ & GQ872418 & Cinnamoyl CoA esters & Cinnamaldehydes & Hu et al., 2011 \\
\hline Cinnamyl alcohol dehydrogenase & CAD & GU937874 & Cinnamyl aldehydes & Cinnamyl alcohols & $\mathrm{Hu}, 2010$ \\
\hline $\begin{array}{l}\text { Hydroxycinnamoyl-CoA shikimate/ } \\
\text { quinate hydroxycinnamoyl transferase }\end{array}$ & $\mathrm{HCT}$ & - & $\begin{array}{l}\text { p-Coumaroyl CoA; } \\
\text { caffeoyl shikimate }\end{array}$ & $\begin{array}{l}\text { p-Coumaroyl shikimate; caffeoyl } \\
\text { CoA }\end{array}$ & Dong et al., 2015 \\
\hline Dirigent protein & DIR1, DIR2 & - & Coniferyl alcohol & Pinoresinol & $\begin{array}{l}\text { Li et al., 2014; } \\
\text { Chen, } 2018\end{array}$ \\
\hline Pinoresinol/lariciresinol reductase & PLR1 & $J F 264893$ & Pinoresinol; lariciresinol & Lariciresinol; secoisolariciresinol & $\begin{array}{l}\text { Xiao et al., 2015; } \\
\text { Xiao et al., } 2021\end{array}$ \\
\hline \multirow[t]{2}{*}{ UDP-glucuronosyltransferase } & UGT71B5a & MW051594 & \multirow{2}{*}{$\begin{array}{l}\text { Pinoresinol; lariciresinol; } \\
\text { secoisolariciresinol; matairesnol }\end{array}$} & \multirow[t]{2}{*}{ Glycosylation products } & \multirow{2}{*}{$\begin{array}{l}\text { Chen et al., } \\
2021 b\end{array}$} \\
\hline & UGT71B5b & MW051595 & & & \\
\hline
\end{tabular}

-; not available

was well explained. Mutagenesis of IPLR1 successfully eliminates the second reaction that converts lariciresinol to secoisolariciresinol, leading to high accumulation of the pharmaceutically valuable compound lariciresinol in E. coli $(997.79 \mathrm{mg} / \mathrm{L})$ (Xiao et al., 2021).

UDP-glucuronosyltransferase (UGT) catalyzes the transfer of the glycosyl group from nucleoside diphosphate-activated sugars (UDP sugars as donors) to a diverse array of secondary metabolites (the acceptors). In I. indigotica, 147 UGTs have been identified and classified into 41 subfamilies. Transcriptome co-expression analysis showed that five genes that encode UGTs (71C1, 71C2, 71D1, 72E3, and 84A4) clustered with DIR2 and DIR3 (the downstream genes involved in the lignan biosynthesis pathway described above) and thus are regarded as lignan glucosyltransferase genes (Chen et al., 2013a). Recently, Chen et al. (2021b) identified two additional UGT-encoding genes (71B5a and 71B5b) that are responsible for glycosylation at the 4-position of pinoresinol. In addition, 71B5a and 71B5b can both catalyze the production of all four compounds downstream of the lignan pathway in I. indigotica from pinoresinol to matairesinol.

The studies on biosynthetic pathways of active compounds provide potential points at which researchers can regulate the features of plants and thus obtain species with ideal accumulation of target compounds. For example, overexpression of $I i \mathrm{PLR} 1$ and $I i \mathrm{C} 3 \mathrm{H}$ leads to a higher accumulation of lariciresinol in hairy roots of $I$. indigotica (Chen et al., 2015; Xiao et al., 2015). Some phytohormones or plant growth regulators such as SA and jasmonic acid (JA) act as conserved elicitors of plant secondary metabolism. Transcriptional factors often play essential roles in their signaling. In addition to activating or suppressing the pathway enzyme genes directly, means to accomplish these assignments are also based on using transcription factors which may influence the flows. Zhang et al. (2016) identified 16 IibHLHs (basic helix-loop-helix) out of the known 78 members of this family in I. indigotica that have a significant positive response to MeJA, and seven of them were highly homologous to known MYCs. A member of APETALA2/ ethylene response factor (AP2/ERF), encoded by Ii049, was identified to be a positive regulator for accumulation of SA, and thus also for lignan content, including lariciresinol, pinoresinol, and secoisolariciresinol, and even for lignins in I. indigotica (Ma et al., 2017a). The transcription factor IiWRKY34 was found not only to regulate lignans but also to have a positive role in root development and stress tolerances (Xiao et al., 2020) (Table 2).

\section{Biosynthesis and regulation of indole alkaloids}

Tryptanthrin, indirubin, and indigo are the main active indole alkaloids in I. indigotica. Indigo and indirubin are known as products formed after fermentation of the aerial parts of this plant, whereas indican is their precursor and 
Table 2 Regulatory genes used for genetic improvement of I. indigotica

\begin{tabular}{|c|c|c|c|c|}
\hline Name & Gene & Gene ID & Function/Pathway & Reference \\
\hline \multirow[t]{2}{*}{ Calcium-dependent protein kinases } & CDPK1 & DQ098651 & \multirow[t]{2}{*}{ Stress-responsive pathway and hormone signaling } & Lu et al., 2006b \\
\hline & CDPK2 & DQ458915 & & Pan et al., 2008 \\
\hline FRUITFUL & FUL & LC321987 & $\begin{array}{l}\text { Multiple reproductive growth stages, including } \\
\text { floral transition, meristem determinacy, floral } \\
\text { organ differentiation and fruit ripening }\end{array}$ & Ma et al., 2017a \\
\hline SHATTERPROOF2 & SHP2 & - & Development of reproductive organs & Lu et al., 2020 \\
\hline \multirow[t]{2}{*}{ SEPALLATA } & SEP1 & LC472304, LC472305 & & Ma et al., 2019 \\
\hline & SEP4 & - & & Pu et al., 2020 \\
\hline Flowering locus $C$ & FLC & - & Flowering related protein & Wang et al., 2018 \\
\hline Later embryogenesis abundant proteins & LEA & AY866484 & - & Lu et al., 2011 \\
\hline GRAS & GRAS & - & Plant growth and development & Zhang et al., 2016c \\
\hline Stomatal density and distribution 1 & SDD1 & DQ407741 & $\begin{array}{l}\text { Regulation of stomatal differentiation and pattern } \\
\text { formation }\end{array}$ & Xiao et al., 2010 \\
\hline \multirow[t]{2}{*}{ AP2/ERF Transcription Factor } & $1 i 049$ & - & Lignan and lignin biosynthesis pathways & Ma et al., 2017 \\
\hline & liERF008 & - & liDIR related factor & Chen, 2018 \\
\hline WRKY Transcription Factor & WRKY34 & MN480620 & $\begin{array}{l}\text { Lignan biosynthesis pathway, stress-responsive } \\
\text { pathway and growth of root }\end{array}$ & Xiao et al., 2020 \\
\hline Insecticidal proteins & Bt and Pta & - & Insect-resistant proteins & Xiao et al., 2012 \\
\hline
\end{tabular}

-; not available

is stored in vacuoles and released after cell death (Chen et al., 2013a; Marcinek et al., 2000; Minami et al., 2000).

The synthesis of indigo, because of its widespread use as a blue dye, has aroused great interest. It is generally believed that indoxyl has an important role in addition to indican in the indigo biosynthetic pathway in I. indigotica. Indoxyl can be synthesized by two paths in the cell. Indoxyl can be biosynthesized from indole, an intermediate compound of the tryptophan pathway (Maier et al., 1990), and the starting point of the process (Xia and Zenk, 1992), in a step catalyzed by a monooxygenase (Fig. 3). Cytochrome P450s (CYPs) and flavincontaining monooxygenases (FMOs) are two major monooxygenase families in plants, and researchers have reported many enzymes from the two families that catalyze this step. For instance, recombinant human CYP2A6 expressed in E. coli can convert indole into indoxyl with NADPH cytochrome P450 reductase (NPR) in vitro (Gillam et al., 1999); this pathway has also been rebuilt successfully in a tobacco cell suspension system (Warzecha et al., 2007). Similarly, CYP102A from Streptomyces cattleya when expressed in E. coli also produces indigo (Kim et al., 2017). Additionally, E. coli expressing bacterial FMOs from Methylophaga sp., Sphingomonas wittichi (Singh et al., 2010), Corynebacterium glutamicum (Ameria et al., 2015), and Nitrincola lacisaponesis (Loncar et al., 2019) can produce indigo.

Alternatively, indoxyl can be synthesized from the degradation of indican as catalyzed by $\beta$-glucosidase (BGL) in Polygonum tinctorium (Minami et al., 1996). Indoxyl is, however, unstable and needs to be converted into indigo in the presence of oxygen or transferred to the next enzyme. The $P$. tinctorium indoxyl $\beta$-glucoside synthase (IGS) most likely carries out this step while using UDP-glucose to recover indican. The identification of protein-protein interactions between $P t$ FMO and PtIGS led to this hypothesis (Inoue et al., 2021).

There are many other kinds of indole alkaloid derivatives. For example, isatans are indoxyl derivatives that differ in the moiety linked to the hydroxyl group of indoxyl-like indican. Isatan A [indoxyl-3-O-(6'-O-malonyl- $\beta$-D-ribohexo-3-ulopyranoside)] and isatan $B$ (indoxyl-3-O- $\beta$-D-ribohexo-3-ulopyranoside) are two major products. The accumulation of isatan $\mathrm{A}$ and the ratio of indican to isatan $\mathrm{B}$ are critical differences between $I$. indigotica and I. tinctoria (Gilbert et al., 2004).

Isatin (indole- $1 \mathrm{H}$-2,3-dione) is obtained from Isatis plants after the oxidation of indigo is complete (Chauhan et al., 2020). Indirubin may be produced as a byproduct of isatin production. Isatin is also an endogenous component of mammalian tissues and body fluids, where it has a variety of activities such as anti-viral, anticorrosive, and transthyretin fibrillogenesis inhibitory activity (Abbas et al., 2013; Gonzalez et al., 2009; Zhang et al., 2014). The metabolic route by which isatin is produced is not clear, although it may result from the synthesis pathway of tryptophan to indole in bacteria followed by oxidation in the liver in humans (Chauhan et al., 2020).

Unfortunately, few of the homologous genes of enzymes that participate in the indigo biosynthesis pathway have been reported for $I$. indigotica. The definitive 


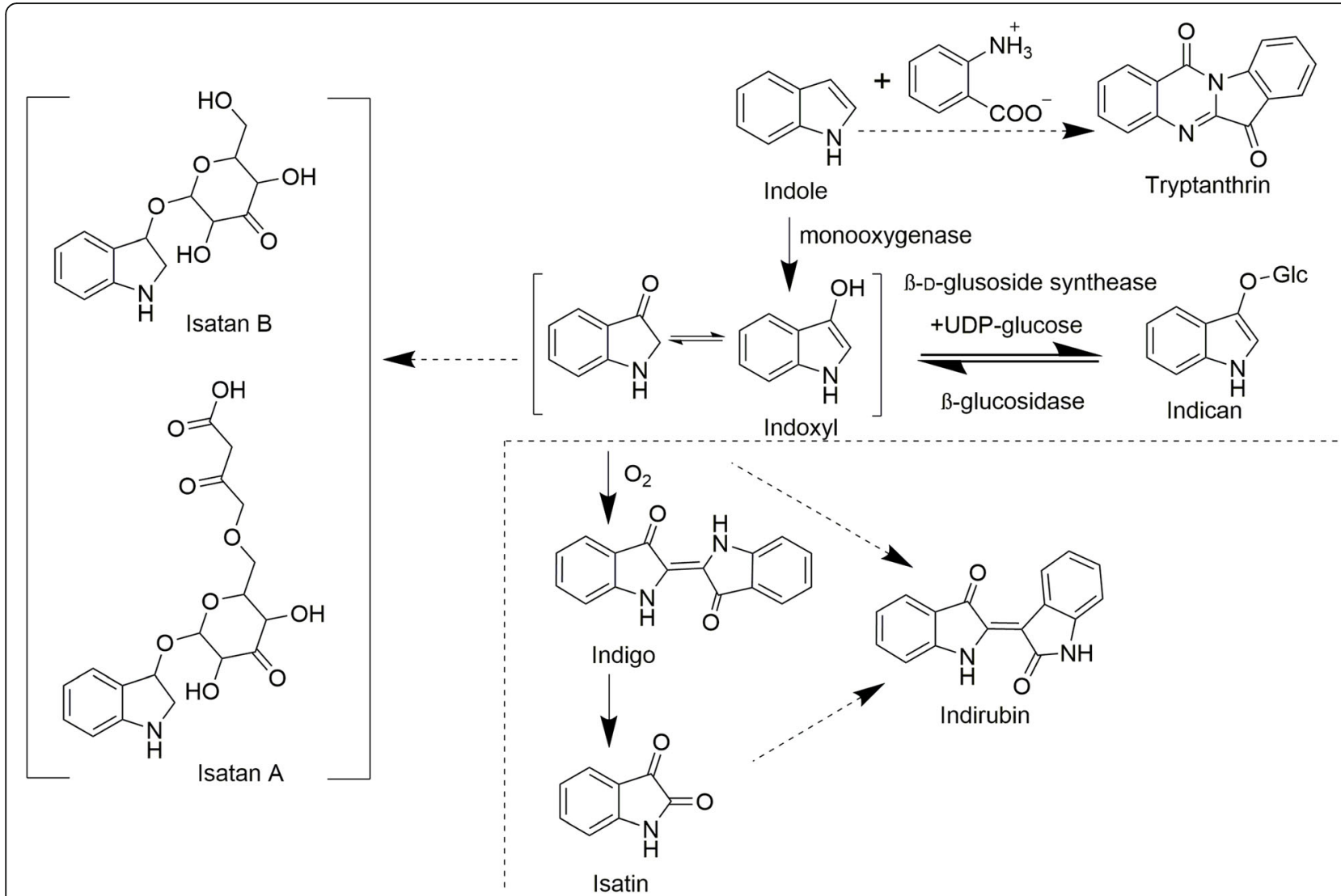

Fig. 3 Biosynthetic pathway of indole alkaloids in I. indigotica. Indoxyl can be biosynthesized from indole or from the degradation of indican; the oxidation or glycosylation reaction make it stable. Indigo, indirubin, and isatin are produced outside of the cells. The figure is adapted from Maier et al. (1990) and Xia and Zenk (1992)

synthesis reaction in this species needs additional investigations.

\section{Biotechnological methods for the improvement of I. indigotica}

Qiao et al. (1989) obtained autotetraploid I. indigotica $(2 \mathrm{n}=28)$ using colchicine treatment after a 5-year selection. This tetraploid has significantly better yield and enhanced resistance to stress relative to its diploid progenitor $(2 \mathrm{n}=14)$.

Researchers used the $A$. thaliana whole-genome Affymetrix gene chip (ATH1) to survey the variation in gene expression between tetraploid and diploid $I$. indigotica. There was a coordinated induction and suppression of 715 and 251 ploidy-responsive genes, respectively, in tetraploid $I$. indigotica that are involved in various developmental, signal transduction, transcriptional regulation, and metabolic pathways ( $\mathrm{Lu}$ et al., 2006a). Several of these genes, including the transcription factor IiWRKY34 (Xiao et al., 2020), the stomatal developmental gene IiSDD1 (Xiao et al., 2010), signal transduction genes IiCPK1 and IiCPK2 (Lu et al., 2006b; Pan et al., 2008), and the lignan biosynthetic pathway gene IiPAL, have been characterized to explore their contribution to the favorable physiological consequences after polyploidization ( $\mathrm{Lu}$ et al., 2006c). In particular, IiWRKY34 has large pleiotropic effects on an array of traits, including yield, lignan biosynthesis, and stress tolerance, which are inferred to have a substantial contribution to the high level of polyploidy vigor of I. indigotica (Xiao et al., 2020) (Table 2). These results may lead to genebased, molecular marker-assisted selection and transformation for the improvement of I. indigotica as an alternative to individually manipulating the component traits using multiple genes with small effects.

Transcriptomic analysis was also used to explore the gene expression changes between tetraploid and diploid I. indigotica. The differentially expressed genes were mainly involved in cell growth, cell wall organization, secondary metabolite biosynthesis, stress response, and photosynthetic pathways (Xiao et al., 2020). Further studies are required to complete the characterization of the mechanisms of autotetraploidy vigor of I. indigotica, which will be helpful for identifying potential targets for genetic improvement. 
Some phenotypic features are specific to I. indigotica compared with other plants in the Cruciferae family. These features may be caused by special genes related to growth and development. The fruits of I. indigotica are referred to as indehiscent silicles, whereas the typical fruits of the Cruciferae family are dehiscent siliques. These morphological variations suggest that I. indigotica undergoes different processes with respect to floral transition and reproductive growth. Some transcription factors related to the development of reproductive organs play important roles in the floral transition process, such as FUL (FRUITFUL), SHP (SHATTERPROOF2), and SEP (SEPALLATA). The IiFUL can affect the development of leaves, florescence meristems, flowers, and fruits (Ma et al., 2017b). Plants that overexpress IiSHP2 bolt earlier, produce leaves that are transformed in shape, have shorter sepals, and have unencapsulated flower buds (Lu et al., 2020). Expression of IiSEP1 and IiSEP4 induces early flowering and reduction of flowers and floral organs in $A$. thaliana, and overexpression of IiSEPs changes the structure of sepals in I. indigotica (Ma et al., 2019; Pu et al., 2020). Negative regulatory factor $I$ FLC (flowering locus $\mathrm{C}$ ) initially decreased and then increased obviously in the development periods from bolting to fruit bearing (Wang et al., 2018). Later embryogenesis abundant (IiLEA) protein did not express in the normal growth conditions in Isatis seedings, its expression level gradually increased with salt or drought stress time, and so may be induced by environmental stress (Lu et al., 2011). Forty-one GRAS genes were identified from $I$. indigotica, which may have crucial roles in diverse plant growth and development (Zhang et al., 2016c).

Currently, tetraploid I. indigotica is widely cultivated to meet the increasing market demand. Despite the greater yield and enhanced stress resistance of these plants, insect damage has remained a substantial problem in its cultivation, affecting both the yield and quality of this medical material. Some attempts have been made to enhance the insect resistance of I. indigotica. Two insecticidal genes (Bt and Pta) were simultaneously introduced into tetraploid $I$. indigotica, resulting in increased protection against both moths and aphids. This was the first attempt to engineer pest control in medicinal plants and offers an efficient molecular breeding strategy for incorporating insect tolerance into such plants (Xiao et al., 2012).

\section{Conclusion and perspectives}

As a well-known indigo-producing and medicinal plant, I. indigotica has been intensively studied over the past 30 years. Great progress has been made in understanding the biosynthesis and metabolic regulation of bioactive compounds such as lignans, indole alkaloids, and their corresponding derivatives. Many key enzymes and transcription factors have been identified and shown to be efficient regulators for the accumulation of lignan and its glycosylated products. It is noteworthy that the biosynthesis pathway of indole alkaloids, including indigo, and the underlying regulatory mechanisms in I. indigotica are not yet fully understood. Although a considerable number of genes have been proposed to be involved in indole alkaloid biosynthesis based on the genome assembly of $I$. indigotica, their function remains to be fully explored. The construction of a "gene-metabolite network" using the available information may help to elucidate this biosynthetic pathway and thus facilitate the process of rationally designing strategies for further improvement of indole alkaloid production.

There are several issues that still need to be addressed;

1. The I. indigotica hairy-root culture system has been widely used in gene functional analyses and metabolic engineering. This system may not, however, be suitable for the characterization of all genes, especially those that function in the aerial parts of $I$. indigotica plants, are involved in plant development, or respond to environmental changes. As an alternative to culture systems, a genetic transformation system has been established for I. indigotica plants. However, the system is not very stable and requires further optimization.

2. A large number of bioactive compounds are produced by $I$. indigotica. The cellular localization and transport and the subcellular compartmentalization and trafficking of these compounds are largely unknown. Meanwhile, metabolic flux results in dynamic changes during the life cycle of all plants. There must be specific mechanisms to control such temporal and spatial profiles. Therefore, exploring the transcriptional regulation mechanism and constructing regulatory networks will be helpful for identifying the specific transcription factors that control specific metabolites.

3. The large-scale production of bioactive compounds by synthetic biology has emerged as an attractive alternative to extracts from I. indigotica. It has been successfully used in the production of bioactive compounds such as lariciresinol. The application of synthetic biology approaches to other bioactive compounds including a series of lignan glycosylated products and indole derivatives is a likely direction for future research.

With the information obtained from sequencing the whole genome of I. indigotica, more functional genes, gene families, and transcriptional regulators will be 
identified, which can be used to improve the quality of $I$. indigotica as an important material for traditional Chinese medicine, to facilitate the production of bioactive compounds through synthetic biology, and to support the research of other medicinally important plants.

\section{Abbreviations}

ABA: abscisic acid; AP2/ERF: APETALA2/ethylene response factor; BCE: Before Common Era; bHLHs: basic helix-loop-helix; CAD: cinnamyl alcohol dehydrogenase; CCOAOMT: caffeoyl COA 3-O-methyltransferase;

CCR: cinnamoyl-CoA reductase; CDPK: calcium-dependent protein kinase; CE: Common Era; CoA: Coenzyme A; CYPs: cytochrome P450s; C3H: coumaric acid 3-hydroxylase; C4H: cinnamate 4-hydroxylase; DIR: dirigent protein; FLC: flowering locus $C$; FMOs: flavin-containing monooxygenases; FUL: FRUITFUL; GA: gibberellic acid; HCT: hydroxycinnamoyl-CoA shikimate/ quinate hydroxycinnamoyl transferase; JA: jasmonic acid; MeJA: methyl jasmonate; NPR: NADPH cytochrome P450 reductase; ORF: open reading frame; PAL: phenylalanine ammonia-lyase; PLR: pinoresinol/lariciresinol reductase; SA: salicylic acid; SEP: SEPALLATA; SHP: SHATTERPROOF2;

SDD1: stomatal density and distribution1; UGT: UDP-glucuronosyltransferase; UV-B: ultraviolet-B; 4CL: 4-coumarate CoA ligase

\section{Acknowledgements}

We apologize to our colleagues whose research works are not properly reviewed here because of space limitation.

\section{Authors' contributions}

W.C. and Y.X. conceived and designed this review article. J.F. and D.H. wrote the draft. Y.Y. prepared the figures. J.C., S.Q., Z.L., X.M., Y.L., R.L., Y.X. and W.C. revised the manuscript. All authors read and approved the final manuscript.

\section{Funding}

This work was supported by the National Natural Science Foundation of China (32170402 and 81874335 to Y.X.; 31872665 to W.C.; 82003897 to J.F.), and the Shanghai local Science and Technology Development Fund Program guided by the Central Government (YDZX20203100002948 to W.C.).

\section{Availability of data and materials}

Not applicable.

\section{Declarations}

Ethics approval and consent to participate

Not applicable.

\section{Consent for publication}

Not applicable.

\section{Competing interests}

The authors declare that they have no competing interests.

\section{Author details}

${ }^{1}$ Research and Development Center of Chinese Medicine Resources and Biotechnology, The Ministry of Education (MOE) Key Laboratory for Standardization of Chinese Medicines, Institute of Chinese Materia Medica, Shanghai University of Traditional Chinese Medicine, Shanghai 201203, China. ${ }^{2}$ Jiangsu Kanion Pharmaceutical Co., Ltd, Jiangsu 222001, Lianyungang, China. ${ }^{3}$ Medical Guarantee Center, Changzheng Hospital, Naval Medical University, Shanghai 200003, China.

Received: 4 August 2021 Accepted: 25 November 2021

Published online: 14 December 2021

\section{References}

Abbas S, Farag A, Ammar Y, Atrees A, Mohamed A, El-Henawy A. Synthesis, characterization, and antiviral activity of novel fluorinated isatin derivatives Monatsh Chem. 2013;144(11):1725-33. https://doi.org/10.1007/s00706-013-1034-3. Ameria S, Jung H, Kim H, Han S, Kim H, Lee J. Characterization of a flavincontaining monooxygenase from Corynebacterium glutamicum and its application to production of indigo and indirubin. Biotechnol Lett. 2015; 37(8):1637-44. https://doi.org/10.1007/s10529-015-1824-2.

Angelini L, Tozzi S, Nasso N. Differences in leaf yield and indigo precursors production in woad (Isatis tinctoria L.) and Chinese woad (Isatis indigotica fort.) genotypes. Field Crop Res. 2007;101(3):285-95. https://doi.org/10.1016/j. fcr.2006.12.004

Anonymous. Shen Nong Ben Cao Jing. Beijing: China Medico-Pharmaceutical Science \& Technology Publishing House; 2008;1:9.

Besseau S, Hoffmann L, Geoffroy P, Lapierre C, Pollet B, Legrand M. Flavonoid accumulation in Arabidopsis repressed in lignin synthesis affects auxin transport and plant growth. Plant Cell. 2007;19(1):148-62. https://doi.org/1 0.1105/tpc.106.044495.

Cao Z, Yang F, Wang J, Gu Z, Lin S, Wang P, et al. Indirubin derivatives as dual inhibitors targeting cyclin-dependent kinase and histone deacetylase for treating Cancer. J Med Chem. 2021;64(20):15280-96. https://doi.org/10.1021/a cs.jmedchem.1 1 01311.

Chauhan G, Pathak D, Ali F, Bhutani R, Kapoor G, Khasimbi S. Advances on synthesis, derivatization and bioactivity of Isatin. Rev Curr Org Synth. 2020; 18(1):37-74. https://doi.org/10.2174/1570179417666200924150907.

Chen J. Ben Cao Meng Quan. Beijing; China press of traditional chinese medicine. 2013;1:27.

Chen J, Dong X, Li Q, Zhou X, Gao S, Chen R, et al. Biosynthesis of the active compounds of Isatis indigotica based on transcriptome sequencing and metabolites profiling. BMC Genomics. 2013;14(1):857. https://doi.org/10.11 86/1471-2164-14-857.

Chen M, Gan L, Lin S, Wang X, Li L, Li Y, et al. Alkaloids from the root of Isatis indigotica. J Nat Prod. 2012;75(6):1167-76. https://doi.org/10.1021/np3002833.

Chen Q, Lan HY, Peng W, Rahman K, Liu QC, Luan X, et al. Isatis indigotica; a review of phytochemistry, pharmacological activities and clinical applications. J Pharm Pharmacol. 2021a;73(9):1137-50. https://doi.org/10.1093/jpp/rgab014.

Chen R. Study on the mechanism of the Dirigent proteins to catalyze lignans in Isatis indigotica. Nav Med Univ. 2018:55-95.

Chen R, Li Q, Tan H, Chen J, Xiao Y, Ma R, et al. Gene-to-metabolite network for biosynthesis of lignans in MeJA-elicited Isatis indigotica hairy root cultures. Front Plant Sci. 2015;6:952. https://doi.org/10.3389/fpls.2015.00952.

Chen X, Chen J, Feng J, Wang Y, Li S, Xiao Y, et al. Tandem UGT71B5s catalyze Lignan glycosylation in Isatis indigotica with substrates promiscuity. Front Plant Sci. 2021 b;12:637695. https://doi.org/10.3389/fpls.2021.637695.

Chen Z, Ben C, Shi Y, Hefei; Anhui Science \& Technology Publishing House. 2004; 3:91-92.

Chinese Pharmacopoeia. Beijing; Chinese pharmacopoeia Commission, China Medical Science and Technology Press. 2020;Part I.

Danz H, Stoyanova S, Wippich P, Brattstrom A, Hamburger M. Identification and isolation of the cyclooxygenase-2 inhibitory principle in Isatis tinctoria. Planta Med. 2001;67(5):411-6. https://doi.org/10.1055/s-2001-15805.

Di P, Hu Y, Xuan H, Xiao Y, Chen W. Characterization and the expression profile of 4-coumarate; CoA ligase (li4CL) from hairy roots of Isatis indigotica. Afr J Pharm Pharmacol. 2012;6(28):2166-75.

Dong H, Yang J, Huang L, Jia J, Tang J. Cloning and expression analysis of a hydroxycinnamoyl-CoA shikimate/quinate hydroxycinnamoyl transferasegene (liHCT) from Isatis indigotica. Zhongguo Zhong Yao Za Zhi. 2015;40(21):4149-54.

European Pharmacopoeia. Strasburg; European Directorate for the Quality of medicines. 2011:3866-3867.

Ghosh R, Chakraborty A, Biswas A, Chowdhuri S. Depicting the inhibitory potential of polyphenols from Isatis indigotica root against the main protease of SARS CoV-2 using computational approaches. J Biomol Struct Dyn. 2020:1-12. https://doi.org/10.1080/07391102.2020.1858164.

Gilbert K, Maule H, Rudolph B, Lewis M, Vandenburg H, Sales E, et al. Quantitative analysis of indigo and indigo precursors in leaves of Isatis spp and Polygonum tinctorium. Biotechnol Prog. 2004;20(4):1289-92. https:/doi.org/1 $0.1021 / \mathrm{bp} 0300624$

Gillam E, Aguinaldo A, Notley L, Kim D, Mundkowski R, Volkov A, et al. Formation of indigo by recombinant mammalian cytochrome $P 450$ Biochem Biophys Res Commun. 1999;265(2):469-72. https://doi.org/10.1 006/bbrc.1999.1702.

Gonzalez A, Quirante J, Nieto J, Almeida M, Saraiva M, Planas A, et al. Isatin derivatives, a novel class of transthyretin fibrillogenesis inhibitors. Bioorg Med Chem Lett. 2009;19(17):5270-3. https://doi.org/10.1016/j.bmcl.2009.03.004.

Grienke U, Zwirchmayr J, Peintner U, Urban E, Zehl M, Schmidtke M, et al. Lanostane triterpenes from Gloeophyllum odoratum and their anti-influenza effects. Planta Med. 2019;85(3):195-202. https://doi.org/10.1055/a-0690-9236. 
Hamburger M. Isatis tinctoria - from the rediscovery of an ancient medicinal plant towards a novel anti-inflammatory phytopharmaceutical. Phytochem Rev. 2002;1(3):333-44. https://doi.org/10.1023/A;1026095608691.

Han B. Shu Ben Cao, Hefei; Anhui Science \& Technology Publishing House. 2005; 6:50.

Han H. Application of JinHuang ointment combine with Radix isatidis granules in children mumps. Chin Foreign Med Treat. 2012;31:117. https://doi.org/10.3 969/j.issn.1674-0742.2012.18.089.

Haruyama T, Nagata K. Anti-influenza virus activity of Ginkgo biloba leaf extracts. J Nat Med. 2013;67(3):636-42. https://doi.org/10.1007/s11418-012-0725-0.

Ho J, Chang H, Lin C, Liu C, Hsieh C, Horng J. Characterization of the antiinfluenza activity of the Chinese herbal plant Paeonia lactiflora. Viruses. 2014; 6(4):1861-75. https://doi.org/10.3390/v6041861.

Hong W, Tang F, Liu J, Liu J, Fang M. In vitro experimental study on the anti dengue virus type II effect of extracts from Folium isatidis. Chin J Mod Drug Appl. 2010;04(20):161-2. https://doi.org/10.3969/j.issn.16739523.2010.20.146

Hsuan S, Chang S, Wang S, Liao T, Jong T, Chien M, et al. The cytotoxicity to leukemia cells and antiviral effects of Isatis indigotica extracts on pseudorabies virus. J Ethnopharmacol. 2009;123(1):61-7. https://doi.org/10.1 016/j.jep.2009.02.028

Hu W, Wu J, Li W. The destructive effect of Radix isatidis extracted by different methods on bacterial endotoxin. Prim J Chin Mater Med. 2003;17(04):60-1. https://doi.org/10.3969/j.issn.1673-6427.2003.04.026.

$\mathrm{Hu}$ Y. Molecular cloning and characterization of five key genes involved in lignan biosynthesis. pathway from Isatis indigotica Fort. Shanghai: Second Military Medical University; 2010:39-79.

Hu Y, Di P, Chen J, Xiao Y, Zhang L, Chen W. Isolation and characterization of a gene encoding cinnamoyl-CoA reductase from Isatis indigotica fort. Mol Biol Rep. 2011;38(3):2075-83. https://doi.org/10.1007/s11033-010-0333-6.

Hu Y, Zhang L, Chen W. Molecular cloning and expression analysis of cinnamic acid 4-hydroxylase gene from Isatis indigotica. Chin Herb Med. 2015;46(1): $101-6$.

Huang D. Study on the chemical characterization of Isatis genus and chemical evolution of Isatis indigotica. Shanghai: Naval Medical University; 2020.

Huang S, Chen C. Textual research on Daqing and Lan. Shanghai J Tradit Chin Med. 1965;10:36.

Inoue S, Morita R, Minami Y. An indigo-producing plant, Polygonum tinctorium, possesses a flavin-containing monooxygenase capable of oxidizing indole. Biochem Bioph Res Commun. 2021;1(534):199-205. https://doi.org/10.1016/j. bbrc.2020.11.112

Jia S, Qi M, Yao S. Beijing; Unity Press 1996;3:86

Kang M, Wu H, Yang Q, Huang L, Hu Q, Ma T, et al. A chromosome-scale genome assembly of Isatis indigotica, an important medicinal plant used in traditional Chinese medicine. Hortic Res. 2020;7(1):18. https://doi.org/10.1038/ s41438-020-0240-5.

Kim H, Jang S, Kim J, et al. Biosynthesis of indigo in Escherichia coli expressing self-sufficient CYP102A from Streptomyces cattleya. Dyes Pigments. 2017;140: 29-35. https://doi.org/10.1016/j.dyepig.2017.01.029.

Lejars $M$, Hajnsdorf $E$. The world of asRNAs in gram-negative and gram-positive bacteria. Biochim Biophys Acta Gene Regul Mech. 1863;2020(2):194489. https://doi.org/10.1016/j.bbagrm.2020.194489.

Li J, Liu Y, Tang J, Xie W. Anti-endotoxic effects of salicylic acid from Radix Isatidis. Chin J Hosp Pharm. 2007;27(10):1349-52.

Li J, Zhou B, Li C, Chen Q, Wang Y, Li Z, et al. Lariciresinol-4-O- $\beta-D-$ glucopyranoside from the root of Isatis indigotica inhibits influenza a virusinduced pro-inflammatory response. J Ethnopharmacol. 2015;174:379-86. https://doi.org/10.1016/j.jep.2015.08.037.

Li Q, Chen J, Xiao Y, Di P, Zhang L, Chen W. The dirigent multigene family in Isatis indigotica; gene discovery and differential transcript abundance. BMC Genomics. 2014;15(1):388. https://doi.org/10.1186/14 71-2164-15-388

Li Y, Yang Y, Zhang G, Li S, Ma X, Chen W, et al. Cloning and Expression Characteristics of Caffeoyl-CoA Oxygen Methyltransferase Gene from Isatis indigotica Fort. Mol Plant Breed. 2021:1-10; Retrieved from https://kns.cnki. net/kcms/detail/46.1068.S.20210707.1557.020.html

Liang Y, Hou H, Li D. Studies on in vitro anticancer activity of tryptanthrin B. Chin Med Herb. 2000;31(7):531-3. doi:10.3321/j.issn:0253-2670.2000.07.029.

Liu Y, Chen M, Guo Q, Lin S, Xu C, Jiang Y, et al. Antiviral glycosidic bisindole alkaloids from the roots of Isatis indigotica. J Asian Nat Prod Res. 2015;17(7): 689-704. https://doi.org/10.1080/10286020.2015.1055729.
Liu Z, Yang Z, Xiao H. Experimental study on the effect of effective monomer of Folium isatidis on respiratory syncytial virus. Lishizhen Med Mater Med Res. 2009;20(08):1977-9. https://doi.org/10.3969/j.issn.10080805.2009.08.070.

Loncar N, Fiorentini F, Bailleul G, Savino S, Romero E, Mattevi A, et al. Characterization of a thermostable flavin-containing monooxygenase from Nitrincola lacisaponensis (NiFMO). Appl Microbiol Biotechnol. 2019;103(4): 1755-64. https://doi.org/10.1007/s00253-018-09579-w.

Lu B. Cloning and functional study of genes related to good quality in tetploid Isatis indigotica. Second Mil Med Univ. 2006:116.

Lu B, Ding R, Zhang L, Yu X, Huang B, Chen W. Molecular cloning and characterization of a novel calcium-dependent protein kinase gene liCPK2 responsive to polyploidy from tetraploid Isatis indigotica. J Biochem Mol Biol. 2006a;39(5):607-17. https://doi.org/10.5483/bmbrep.2006.39.5.607.

Lu B, Du Z, Ding R, Zhang L, Yu X, Liu C, et al. Cloning and characterization of a differentially expressed phenylalanine Ammonialyase gene (liPAL) after genome duplication from tetraploid Isatis indigotica fort. J Integr Plant Biol. 2006c;48(12):1439-49. https://doi.org/10.1111/j.1744-7909.2006.00363.x.

Lu B, Pan X, Zhang L, Huang B. A genome-wide comparison of genes responsive to autopolyploidy in Isatis indigotica using Arabidopsis thaliana Affymetrix genechips. Plant Mol Biol Rep. 2006b;24(2):197-204. https://doi.org/10.1007/ BF02914058.

Lu H, Liu J, Deng R, Song J. Preparative isolation and purification of indigo and indirubin from folium isatidis by high-speed counter-current chromatography. Phytochem Anal. 2012;23(6):637-41. https://doi.org/10.1002/pca.2366.

Lu M, Li D, Pu Z, Ma Y, Huang X, Xu Z. Ectopic expression of liSHP2 from Isatis indigotica fortune, a PLE-lineage MADS-box gene, influences leaf, floral organ and silique morphology in Arabidopsis thaliana. Physiol Mol Biol Plants. 2020; 26(2):379-89. https://doi.org/10.1007/s12298-019-00745-6.

Lu S, Jia R, Du F, Liu Z, Yu J. Cloning and expression analysis under stress of liLEA gene in Isatis indigotica. Acta Bot Boreal-Occident Sin. 2011;31(3):511-6.

Luo Z, Liu L, Wang X, Li W, Jie C, Chen H, et al. Epigoitrin, an alkaloid from Isatis indigotica, reduces $\mathrm{H} 1 \mathrm{~N} 1$ infection in stress-induced susceptible model in vivo and in vitro. Front Pharmacol. 2019;10:78. https://doi.org/10.3389/fpha r.2019.00078

Ma J. Analysis of modern pharmacology and clinical application of Radix isatidis. Chin Health Standard Manag. 2014;5:65-6. https://doi.org/10.3969/J.ISSN.1 674-9316.2014.06.038

Ma R, Liu Q, Xiao Y, Zhang L, Li Q, Yin J, et al. The phenylalanine ammonia-lyase gene family in Isatis indigotica fort:; molecular cloning, characterization, and expression analysis. Chin J Nat Med. 2016;14(11):801-12. https://doi.org/10.1 016/s1875-5364(16)30097-8.

Ma R, Xiao Y, Lv Z, Tan H, Chen R, Li Q, et al. AP2/ERF transcription factor, li049, Positively Regulates Lignan Biosynthesis in Isatis indigotica through Activating Salicylic Acid Signaling and Lignan/Lignin Pathway Genes. Front Plant Sci. 2017a;8:1361. https://doi.org/10.3389/fpls.2017.01361.

Ma W. An observation on therapeutic efficacy of Isatis indigotica fort co granules in patients with viral myocarditis. Pract J Card Cerebr Pneumal Vasc Dis. 2003;11(03):135-8. https://doi.org/10.3969/j.issn.1008-5971.2003.03.003.

Ma Y, Li D, Zhang L, Li Q, Yao J, Ma Z, et al. Ectopic expression of liFUL isolated from Isatis indigotica could change the reproductive growth of Arabidopsis thaliana. Plant Physiol Biochem. 2017b;121:140-52. https://doi.org/10.1016/j. plaphy.2017.10.014

Ma Y, Pu Z, Zhang L, Lu M, Zhu Y, Hao C, et al. A SEPALLATA1-like gene of Isatis indigotica Fort. regulates flowering time and specifies floral organs. Gene. 2019;713:143974. https://doi.org/10.1016/j.gene.2019.143974.

Maier W, Schumann B, GroGer D. Biosynthesis of indoxyl derivatives in Isatis tinctoria and Polygonum tinctorium. Phytochemistry. 1990;29(3):817-9. https://doi.org/10.1016/0031-9422(90)80025-C.

Marcinek H, Weyler W, Deus-Neumann B, Zenk M. Indoxyl-UDPGglucosyltransferase from Baphicacanthus cusia. Phytochemistry. 2000;53(2): 201-7. https://doi.org/10.1016/s0031-9422(99)00430-6.

Meng L, Guo Q, Liu Y, Chen M, Li Y, Jiang J, et al. Indole alkaloid sulfonic acids from an aqueous extract of Isatis indigotica roots and their antiviral activity. Acta Pharm Sin B. 2017a;7(3):334-41. https://doi.org/10.1016/j.apsb.2017.04.003.

Meng L, Guo Q, Xu C, Zhu C, Liu Y, Chen M, et al. Diglycosidic indole alkaloid derivatives from an aqueous extract of Isatis indigotica roots. J Asian Nat Prod Res. 2017b;19(6):529-40. https://doi.org/10.1080/10286020.2017.1320547.

Minami Y, Kanafuji T, Miura K. Purification and characterization of a $\beta$-glucosidase from Polygonum tinctorium, which catalyzes preferentially the hydrolysis of Indican. J Agric Chem Soc Jpn. 1996;60(1):147-9. 
Minami Y, Nishimura O, Hara-Nishimura I, Nishimura M, Matsubara H. Tissue and intracellular localization of indican and the purification and characterization of indican synthase from indigo plants. Plant Cell Physiol. 2000;41(2):218-25. https://doi.org/10.1093/pcp/41.2.218

Pan $X$, Ying X, Wang Z, Lei Z, Tang K. Tetraploids Isatis indigotica are more responsive and adaptable to stresses than the diploid progenitor based on changes in expression patterns of a cold inducible liCPK1. Biologia. 2008; 63(4):535-41. https://doi.org/10.2478/s11756-008-0094-Z.

Pu Z, Ma Y, Lu M, Ma Y, Xu Z. Cloning of a SEPALLATA4-like gene (liSEP4) in Isatis indigotica fortune and characterization of its function in Arabidopsis thaliana. Plant Physiol Biochem. 2020;154:229-37. https://doi.org/10.1016/j.plaphy.202 0.05.031.

Qiao C. Study on the identification of Isatis indigotica Fort. and Isatis tinctoria L. J Syst Evol. 1984;22:3.

Qiao C, Cui X. Polyploid breeding of Isatis indigotica Fort. Chin Pharmaceut Bull. 1982;17(1):54

Qiao C, Wu M, Dai F, Cui X, Li L. Studies on Polyploid breeding of Isatis indigotica fort. Acta Bot Sin. 1989;31(9):678-83.

Shi G, Zhang J. Experimental study on the pharmacological effect of ethanol precipitate of Folium isatidis. J Henan Univ Chin Med. 2006;21(4):15-6. https://doi.org/10.16368/j.lssn.1674-8999.2006.04.009.

Singh A, Singh Chauhan N, Thulasiram H, Taneja V, Sharma R. Identification of two flavin monooxygenases from an effluent treatment plant sludge metagenomic library. Bioresour Technol. 2010;101(21):8481-4. https://doi. org/10.1016/j.biortech.2010.06.025.

Speranza J, Miceli N, Taviano M, Ragusa S, Kwiecien I, Szopa A, et al. Isatis tinctoria L. (Woad); A Review of its Botany, Ethnobotanical Uses, Phytochemistry, Biological Activities, and Biotechnological Studies. Plants (Basel). 2020;9(3):298. https://doi.org/10.3390/plants9030298.

Su S, Ben C, Tu J. Hefei; Anhui Science \& Technology Publishing House. 1994;4:110.

Sun Z, Pang X. A high degree of genetic diversity is revealed in Isatis spp. (dyer's woad) by amplified fragment length polymorphism (AFLP). Theor Appl Genet. 2002;104(6-7):1150-6. https://doi.org/10.1007/s00122-001-0863-3.

Sun Z, Pang X. Discussion on the botanical origin of Isatidis radix and Isatidis folium based on DNA barcoding. Yao Xue Xue Bao. 2013;48(12):1850-5.

Tang J, Shi C, Fang J, Wang W, Liu Y. Effects of Radix Isatidis on the level of LPO and SOD in serum of endotoxin-induced DIC rabbits. Her Med. 2004;23(1):45. https://doi.org/10.3870/j.issn.1004-0781.2004.01.003.

Tao H, Ming Y, Bie L. Beijing; China medico-pharmaceutical science \& technology publishing house. 2013;1:46.

Teng J. Textual research on Qingdai and Lan. Chin Tradit Herb Drugs. 1996;27(2): 110-112.

Wang $H, M a Y$, Zhou W. Toxicity test of Isatis polysaccharide and its effect on immune system of mice. J Mianyang Normal Univ. 2012;31(2)75-80. https:// doi.org/10.3969/j.issn.1672-612X.2012.02.021.

Wang L, Bai Y, Zhang X, Liu J, Tang X, Yang J, et al. Cloning and expression analysis of flowering inhibitor liFLC in Isatis indigotica fort. Mol Plant Breed. 2018;16(1):1-8.

Wang $X$, Zhou T. Textual research on scientific name of Isatis indigotica Fort. Chin Tradit Herb Drugs. 1982;13(11):37-8

Wang Y, Jin Y, Chen Z, Chen S, Zhan Z. Textual research on medicinal blue herbs. Chin J Chin Mater Med. 2020;45(23):5819-28.

Warzecha H, Frank A, Peer M, Gillam E, Guengerich F, Unger M. Formation of the indigo precursor indican in genetically engineered tobacco plants and cell cultures. Plant Biotechnol J. 2007;5(1):185-91. https://doi.org/10.1111/j.14677652.2006.00231.x.

Wu P, Wang J. Textual research on Daqing, Banlangen and Qingdai. Journal of Shanghai Research Institute of Traditional Chinese Medicine in Shanghai University of Traditional Chinese medicine. 1996;1.

Xia Z, Zenk M. Biosynthesis of indigo precursors in higher plants. Phytochemistry. 1992;31(8):2695-7. https://doi.org/10.1016/0031-9422(92)83613-4.

Xiao Y, Feng J, Li Q, Zhou Y, Bu Q, Zhou J, et al. liWRKY34 positively regulates yield, lignan biosynthesis and stress tolerance in Isatis indigotica. Acta Pharm Sin B. 2020;10(12):2417-32. https://doi.org/10.1016/j.apsb.2019.12.020.

Xiao Y, Ji Q, Gao S, Tan H, Chen R, Li Q, et al. Combined transcriptome and metabolite profiling reveals that liPLR1 plays an important role in lariciresinol accumulation in Isatis indigotica. J Exp Bot. 2015;66(20):6259-71. https://doi. org/10.1093/jxb/erv333.

Xiao Y, Shao K, Zhou J, Wang L, Ma X, Wu D, et al. Structure-based engineering of substrate specificity for pinoresinol-lariciresinol reductases. Nat Commun. 2021;12(1):2828. https://doi.org/10.1038/s41467-021-23095-y.
Xiao Y, Wang K, Ding R, Zhang H, Di P, Chen J, et al. Transgenic tetraploid Isatis indigotica expressing Bt Cry1 Ac and Pinellia ternata agglutinin showed enhanced resistance to moths and aphids. Mol Biol Rep. 2012;39(1):485-91. https://doi.org/10.1007/s11033-011-0762-X.

Xiao Y, Yu X, Chen J, Di P, Chen W, Zhang L. liSDD1, a gene responsive to autopolyploidy and environmental factors in Isatis indigotica. Mol Biol Rep. 2010;37(2):987-94. https://doi.org/10.1007/s11033-009-9776-Z.

Xu Y, Liu J, Zhao B, Wang D, Fan Y, Hu Y, et al. Effect of acid of radix isatidis alkaloid on adsorption and release of Newcastle disease virus. J Nanjing Agric Univ. 2010;33:90-4. https://doi.org/10.7685/j.issn.1000-2030.2010.06.017.

Xuan $\mathrm{H}$. Functional study of five genes on the lignan biosynthesis pathway in Isatis indigotica. Heilongjiang: Jiamusi University; 2012.

Yang S, Ben C, Shu GY. Shanxi; Shanxi science and technology Publisher. 2009;9: 260-2.

Yang Z, Wang Y, Zheng Z, Zhao S, Zhao J, Lin Q, et al. Antiviral activity of Isatis indigotica root-derived clemastanin $B$ against human and avian influenza a and B viruses in vitro. Int J Mol Med. 2013;31(4):867-73. https://doi.org/10.3 892/ijmm.2013.1274.

Yang Z, Wang Y, Zhong S, Zhao S, Zeng X, Mo Z, et al. In vitro inhibition of influenza virus infection by a crude extract from Isatis indigotica root resulting in the prevention of viral attachment. Mol Med Rep. 2012;5(3):7939. https://doi.org/10.3892/mmr.2011.709.

Ye W, Li X, Cheng J. Screening of eleven chemical constituents from Radix isatidis for antiviral activity. Afr J Pharm Pharmacol. 2011;5(16):1932-6.

Yong J, Aisa H. Chemlnform abstract; chemical modification of Rupestonic acid and preliminarily in vitro antiviral activity against influenza $A 3$ and $B$ viruses. ChemInform. 2011;42(36):1293-7. https://doi.org/10.1002/chin.201136179.

Zeng Q, Luo C, Cho J, Lai D, Shen X, Zhang X, et al. Tryptanthrin exerts antibreast cancer effects both in vitro and in vivo through modulating the inflammatory tumor microenvironment. Acta Pharma. 2021;71(2):245-66. https://doi.org/10.2478/acph-2021-0020.

Zhang D, Li J, Ruan D, Chen Z, Zhu W, Shi Y, et al. Lignans from Isatis indigotica roots and their inhibitory effects on nitric oxide production. Fitoterapia. 2019a;137:104189. https://doi.org/10.1016/j.fitote.2019.104189.

Zhang D, Shi Y, Xu R, Du K, Guo F, Chen K, et al. Alkaloid Enantiomers from the Roots of Isatis indigotica. Molecules. 2019b;24(17):3140. https://doi.org/10.33 90/molecules 24173140

Zhang H, Dai H, Hanson P, Li H, Guo H, Ye X, et al. Antiviral Activity of an Isatin Derivative via Induction of PERK-Nrf2-Mediated Suppression of CapIndependent Translation. ACS Chem Biol. 2014;9(4):1015-24. https://doi.org/1 $0.1021 / \mathrm{cb} 400775 z$.

Zhang L, Chen J, Li Q, Chen W. Transcriptome-wide analysis of basic helix-loophelix transcription factors in Isatis indigotica and their methyl jasmonate responsive expression profiling. Gene. 2016a;576(1):150-9. https://doi.org/10.1 016/j.gene.2015.09.083.

Zhang L, Chen J, Zhou X, Chen X, Li Q, Tan H, et al. Dynamic metabolic and transcriptomic profiling of methyl jasmonate-treated hairy roots reveals synthetic characters and regulators of lignan biosynthesis in Isatis indigotica fort. Plant Biotechnol J. 2016b;14(12):2217-27. https://doi.org/10.1111/pbi.12576.

Zhang L, Li Q, Chen J, Chen W. Computational identification and systematic classification of novel GRAS genes in Isatis indigotica. Chin J Nat Med. 2016c; 14(3):161-76. https://doi.org/10.1016/S1875-5364(16)30013-9.

Zhang Z, Morris-Natschke S, Cheng Y, Lee K, Li R. Development of anti-influenza agents from natural products. Med Res Rev. 2020;40(6):2290-338. https://doi. org/10.1002/med.21707.

Zhao J, Aisa H. Synthesis and anti-influenza activity of aminoalkyl rupestonates. Bioorg Med Chem Lett. 2012;22(6):2321-5. https://doi.org/10.1016/j.bmcl.2 012.01.056.

Zhao Y, Wang J, Shan L, Jin C, Ma L, Xiao X. Effect of Radix isatidis polysaccharides on immunological function and expression of immune related cytokines in mice. Chin J Integr Med. 2008;14(3):207-11. https://doi. org/10.1007/s11655-008-0207-2.

Zhou L, Yuan B, Su X. Antimicrobial activity in vitro and antiendotoxin activity of the compound Isatis indigotica Fort.injection. J Shenyang Pharm Univ. 2006; 23(4):247-50. doi: 10.3969/j.issn.1006-2858.2006.04.014.

Zhu S, Chai X, Kuang R. Pharmacodynamics study on Fufangdaqinye Mixture. Chin Tradit Patent Med. 2004;26(11):909-12.

\section{Publisher's Note}

Springer Nature remains neutral with regard to jurisdictional claims in published maps and institutional affiliations. 